\title{
Blocking in Rabbit Eyeblink Conditioning Is Not Due to Learned Inattention: Indirect Support for an Error Correction Mechanism of Blocking
}

\author{
M. Todd Allen, Yahaira Padilla, and Mark A. Gluck \\ Center for Molecular and Behavioral Neuroscience \\ Rutgers University, Newark, New Jersey
}

\begin{abstract}
Blocking is a classical conditioning task in which prior training to one cue such as a tone reduces learning about a second cue such as a light, when subsequently trained as a tonelight compound. Blocking has been theorized to come about through a US-modulated error correction mechanism by Rescorla \& Wagner (1972) as well as through a mechanism of learned inattention as theorized by Mackintosh (1973). In the case of eyeblink conditioning, an error correction mechanism has been hypothesized to take place in the cerebellum while some form of inattention has been hypothesized to take place in the hippocampal region. The hypothesis we are testing is whether the mechanism of learned inattention is involved in blocking in rabbit eyeblink conditioning. If blocking in eyeblink conditioning is produced by a mechanism of learned inattention, then training to a previously blocked cue should be slower than training to that cue in a naïve animal. Rabbits that had received tone training followed by tone-light training exhibited blocking. Rabbits that had been previously blocked to the light acquired conditioned responses to the light at the same rate as naïve rabbits. This finding failed to support the hypothesis that blocking in rabbit eyeblink conditioning is due to learned inattention, but does support the Rescorla-Wagner mechanism of error correction. The present finding along with previous work on error correction mechanism in the cerebellar-brainstem circuit (Kim et al., 1998) lend support to the theory that blocking, at least in rabbit eyeblink conditioning, seems to be due to an error correction mechanism rather than a learned inattention mechanism.
\end{abstract}

The Kamin (1969) blocking effect is a classical conditioning task that has been the focus of many empirical tests and theories. Blocking is a conditioning task in which prior training to one cue (e.g., a tone) reduces learning about a second cue (e.g., a light) in subsequent tone-light compound training as compared to controls that were only trained to the tone-light compound. Evidence of blocking is usually demonstrated by either test trials of the second cue alone or by training the second cue to test how blocking affected subsequent acquisition.

Mechanisms such as error correction (Rescorla \& Wagner, 1972) and learned inattention (Mackintosh, 1973) have been put forth as being responsible for blocking. An error correction mechanism of blocking assumes that there is no learning to the added cue due to the US being predicted by the previously trained cue. A learned inattention mechanism

Address for Correspondence: M. Todd Allen, Ph.D., Psychology Dept., College of Charleston, Charleston, SC 29412 Ph\# 843-953-6362 Fax\# 843-953-7151 E-mail: allenm@ cofc.edu

Integrative Physiological \& Behavioral Science, October-December 2002, Vol. 37, No. 4, 254-264. 
assumes that there is a learned inattention to the added cue due to its redundancy to the previously trained cue. Therefore, these two mechanisms differ in how they predict learning to a previously blocked cue will progress. Error correction predicts that learning to a previously blocked cue will occur as if the subject were naïve to the cue. Conversely, learned inattention predicts that learning to a previously blocked cue will begin at a slower rate due to the cue previously being inattended to.

These mechanisms have been mapped to different brain regions for particular forms of classical conditioning. In the case of eyeblink conditioning, error correction has been mapped to the cerebellum while learned inattention has been mapped to the hippocampus. Classical eyeblink conditioning is an associative learning paradigm in which a neutral stimulus such as a tone or light (the conditioned stimulus or CS) is paired with a responseevoking stimulus such as a corneal air puff (the unconditioned stimulus or US). Initially, the CS elicits no behavioral response while the US elicits a protective eyeblink response. By repeatedly pairing the CS followed by the US, the CS alone comes to elicit an anticipatory eyeblink response (the conditioned response or CR). For a complete review of this behavioral literature, see Gormezano et al. (1983). Many researchers have demonstrated this blocking phenomenon in classical eyeblink conditioning in rabbits (Marchant and Moore, 1973; Kinkaide, 1974; Solomon, 1977; Kim Krupa \& Thompson, 1998) as well as humans (Martin \& Levey, 1991).

Eyeblink conditioning involves interactions between the cerebellar and hippocampal systems based on task demands (Allen et al., 2001). Studies have shown that the cerebellum, specifically, the deep interpositus nucleus, is the essential substrate for the acquisition, expression, and retention of the classically conditioned eyeblink response (for review see: Anderson \& Steinmetz, 1994; Kim \& Thompson, 1997). The learning mechanism hypothesized to occur in the cerebellar system is error correction (Gluck et al., 2001; Kim et al., 1998). It has been proposed that an inhibitory feedback pathway between the cerebellum and the US input pathway via the inferior olive performs the Rescorla-Wagner (1972) error correction function (Donegan \& Wagner, 1987; Donegan, Gluck, \& Thompson, 1989; Gluck, Myers \& Thompson, 1994; Gluck et al., 2001). In the Rescorla-Wagner (1972) rule, strengthening of the CS-US association is driven by errors in the prediction of the US.

As learning to predict the US improves CRs are produced that precede the US. As CRs develop, the error in US prediction that normally drives learning decreases. Electrophysiological recordings have demonstrated that as eyeblink CRs develop, the US-related activity in the inferior olive decreases (Sears \& Steinmetz, 1991). In addition, Kim et al. (1998) found that blocking in eyeblink conditioning was disrupted by a pharmacological manipulation of a inhibitory feedback pathway from the cerebellum back to the US input pathway in the inferior olive. Manipulations of this feedback pathway between the cerebellum and the inferior olive provide evidence that a neural substrate can be shown to operate on the same principles as the Rescorla Wagner rule of error correction.

One learning mechanism hypothesized to occur in the hippocampal system during some eyeblink conditioning tasks is based on inhibition or inattention and is comparable to the learned inattention mechanism of Mackintosh (1973). There is a long history of hippocampal theories involving different forms of inhibition and learned inattention (Douglas, 1967; Douglas \& Pribram, 1966; Chan et al., 2001). In eyeblink conditioning, Solomon (1980) proposed that the hippocampus acts as an inhibitor of attention that served to tune out irrelevant stimuli during classical conditioning. In the case of blocking, the added redundant cue is an irrelevant stimulus. This mechanism is similar to Mackintosh's mechanism 
of learning inattention. Therefore, in eyeblink conditioning the hippocampus may alter attention to inhibit responding to irrelevant stimuli such as the novel but redundant cue added during blocking training.

A computational model of eyeblink conditioning put forth by Gluck \& Myers (1993) hypothesized that the hippocampus plays a role in altering stimulus representations during learning, which has been viewed as a form of CS modulation (Allen et al., 2001). These models are based on the idea that cerebellar and hippocampal systems interact. It is possible that both error correction and some hippocampal inhibition mechanism are interacting to produce blocking.

The idea that blocking could be determined by both a hippocampal and a non-hippocampal mechanism has been put forth by Holland (1997). Holland theorized that blocking could involve both an error correction mechanism and a hippocampal mechanism that reduced CS associability. Baxter et al. (1999) presented evidence from an appetitive conditioning task in rats that had undergone hippocampal disruption through a lesion of the cholinergic inputs from the medial septum that supported this theory. They found that blocking was evident as tested by test trials of the blocked cue, but that there was no subsequent slower learning to the blocked cue as compared to non-blocked controls. This finding indicates that, at least for appetitive conditioning, a hippocampal mechanism like learned inattention operates in conjunction with a hippocampal independent mechanism of error correction.

We were interested in determining if these two mechanisms might be interacting in the production of blocking in rabbit eyeblink conditioning. Recent work has supported the idea of an error correction mechanism as working in the cerebellar system (Kim et al., 1998). In addition, some mechanisms of inhibition or inattention have been hypothesized as acting in the hippocampal region (Solomon, 1980). However, the specific mechanism of learned inattention as proposed by Mackintosh \& Turner (1971) has not been tested in rabbit eyeblink conditioning to date.

In this paper we report the results from a test of learned inattention with blocking in rabbit eyeblink conditioning using a tone and a light. We will address the issue of learned inattention by comparing the learning to a blocked cue (a light) to learning about the same cue in naïve rabbits. If blocking is due to error correction as predicted by the RescorlaWagner (1972) rule, then there should no learning to a blocked cue during compound tonelight training. Training to the previously blocked cue should then begin as if naïve and be learned at the same rate. However, if blocking is due to a mechanism of learned inattention as proposed by Mackintosh \& Turner (1971), then training to a previously blocked light should be slower than normal learning in naïve rabbits due to having to overcome this previously learned inattention to the redundant cue. Therefore, the learned inattention mechanism predicts learning to the blocked light should be slower than learning to the light in naïve rabbits. Through this test of possible mechanisms of blocking, we hope to determine whether the mechanism of learned inattention is involved in blocking in eyeblink conditioning.

\section{Methods}

Subjects: Eighteen male rabbits (weight over $2.1 \mathrm{~kg}$ ) were used in this experiment. The rabbits were purchased from Covance Laboratories. The rabbits were housed in individual cages in the Rutgers University Animal Facility, Newark, New Jersey. They were given free access to food and water. They were maintained on a $12 \mathrm{hr}$ light /dark cycle with light 
on at 7:00 a.m. All testing occurred between 8 a.m. and 6 p.m. All procedures with the rabbits were approved by the Rutgers IACAUC (Rutgers animal protocol \# 96-010).

Materials: The rabbits were restrained in plexi-glass restraint boxes in individual conditioning chambers. Each chamber contained a speaker, air hose assembly, and eyeblink detection system. Presentations of the stimuli and recording of behavioral responses were controlled by a PC computer. The computer housed an interface board (Keithley Metrabyte, Tujunga, CA), which triggered a set of relays that controlled the presentation of the tone CS and air puff US. The software that controls the interface was designed by Chen \& Steinmetz (1998).

Eyeblinks were monitored with an opto-electronic sensor that consisted of a light emitting diode (LED) and a phototransistor (for technical details see Thompson et al., 1994). The LED emitted a beam of infrared light that was reflected off the cornea, and the reflectance of this beam was converted to a DC voltage by a phototransistor. The eyeblink signal was filtered (between $.1 \mathrm{~Hz}$ and $1 \mathrm{KHz}$ ) and amplified (100x) by a differential AC amplifier (A-M Systems, Everett, WA).

When the rabbit closed its eye, the reflectance of the infrared beam changed and was recorded as an eyeblink. Any movement greater than $0.5 \mathrm{~mm}$ during the preCS period caused the training trial to be discarded from analysis. A conditioned response (CR) was scored if movement of greater than $0.5 \mathrm{~mm}$ was recorded in the CS period (i.e., the time between the CS and US onsets). An unconditioned response (UR) was scored if movement of greater than $0.5 \mathrm{~mm}$ was recorded following the US presentation. Each trial's behavioral record was displayed on the computer screen. The computer analyzed the behavioral data and delivered the data for each block of 50 trials.

Stimuli: The CS was either a $450 \mathrm{~ms}, 90 \mathrm{~dB}, 1000 \mathrm{~Hz}$ tone or a $450 \mathrm{~ms}, 12 \mathrm{~V}$ light delivered from a panel of 4 bulbs directly in front of the rabbit's face. The US was a 50 ms, 4 psi corneal air puff delivered via a rubber hose attached to the eyeblink detector assembly and aimed at the rabbit's cornea. All training trials were paired presentations in which the CS onset was $400 \mathrm{~ms}$ prior to the US onset and the CS and US co-terminated. The inter-trial interval between training trials was a pseudo-random number that averaged $30 \mathrm{~s}$ and ranged from 25 to 35 seconds. All training was done in the darkened chambers with a red light outside in the conditioning room.

\section{Design and Procedure}

Adaptation: Prior to acquisition, each rabbit was adapted to the conditioning chamber and restraint box for two daily sessions. For the first session of adaptation, the rabbit was placed in the restraint box in the conditioning chamber for a half-hour. On the second session of adaptation, the rabbit was placed in the restraint box in the conditioning chamber for 45 minutes with the eyeblink detector aimed at its cornea. Rabbits were equally divided between a blocking group, a control group, and a naïve group.

Blocking and Control Acquisition: Blocking and control acquisition consisted of four phases of training. Phase 1 consisted of 700 trials over seven consecutive daily sessions. The blocking group received tone-air puff training in Phase 1 while the control group received 700 blank trials with no stimuli presented. Phase 2 consisted of 500 trials of tonelight air puff training over five consecutive daily sessions for both the blocking and control groups. Phase 3 consisted of 30 test light alone trials for both the blocking and control groups. Phase 4 consisted of 500 light-air puff trials over five consecutive daily sessions for both the blocking and control groups. 
Naive Acquisition: Rabbits in the naive group received 500 trials of light and air puff trials. The data from these rabbits was compared to the data from blocking and control rabbits for phase 4 to test how blocking affected the acquisition of CRs to the light.

Following light training, all rabbits were euthanized by an overdose injection (IV) of pentobarbital via the marginal ear vein.

Data Analysis: Behavioral results in the form of percentage of conditioned responses (\%CR) for all phases were analyzed with a repeated measures ANOVA in 50 trial blocks with the exception of the data from phase 3 , which were analyzed as a single block of 30 trials with an independent measures t-test.

\section{Results}

One rabbit in the blocking group failed to acquire CRs to the light during phase 4. This is highly unusual since all other rabbits eventually exhibited CRs to the light. This failure was not normal for the blocking effect since all animals should have eventually learned to the blocked cue. This individual subject's failure to learn to the visual cue may have been due to some sensory deficit. Therefore, its behavioral data for all phases of conditioning was excluded from data analysis. All other rabbits were able to acquire CRs to the tone, light, and tone-light compound.

For the tone training in Phase 1, all rabbits in the blocking group exhibited more conditioned responses as training progressed across sessions $(\mathrm{F}(9,81)=5.368, \mathrm{p}<.001)$, as shown in Figure 1A. Rabbits in the control group did not receive any stimuli during phase one and produced no data and are not graphed in Figure 1A.

For the tone-light training in Phase 2, all rabbits exhibited more conditioned responses to the tone-light compound as training progressed across sessions $(\mathrm{F}(9,81)=14.051, \mathrm{p}<$ .001). Rabbits in the blocking group exhibited significantly more conditioned responses than those in the control group $(\mathrm{F}(1,9)=.919, \mathrm{p}<.005)$, as shown in Figure $1 \mathrm{~B}$. There was a significant interaction $(\mathrm{F}(9,81)=14.051, \mathrm{p}<.001)$ such that early in phase 2 , blocking rabbits exhibited more conditioned responses than the controls, but this difference disappeared later on in Phase 2. By the end of tone-light training, all rabbits were exhibiting asymptotic levels of conditioned responses to the tone-light compound.

One interesting effect observed in phase 2 was that the acquisition of CRs to the tonelight compound by the control group appeared to be facilitated as compared to the learning of the blocking group to the tone in phase 1. Analysis of the data for the first 150 trials of phase 1 and 2 indicated that control rabbits exhibited more CRs to the tone-light compound than did blocking rabbits to the tone alone $(\mathrm{F}(1,9)=8.009, \mathrm{p}<.02)$.

In Phase 3, the test for blocking, rabbits in the control group exhibited significantly more conditioned responses to light alone test trials than did those in the blocking group ( $t$ $(17)=-3.207, p<.01)$, as shown in Figure $1 \mathrm{C}$.

For the light training in phase 4 , all rabbits exhibited significantly more conditioned responses to the light as training progressed across sessions, $(\mathrm{F}(9,81)=15.044, \mathrm{p}<.001)$. Rabbits in the control group exhibited significantly more conditioned responses than did those in the blocking group $(\mathrm{F}(1,9)=7.907, \mathrm{p}<.05)$, as shown in Figure 1D. There was also a significant interaction of sessions and groups $(\mathrm{F}(9,81)=2.833, \mathrm{p}<.01)$, such that early in phase 4 there were significant differences between the blocking and control groups, but these differences disappeared by the middle of training to the light.

To determine whether learning to the light during the tone-light training in phase 2 was actively inhibited through a process of learned inattention, the phase 4 data of the blocking 

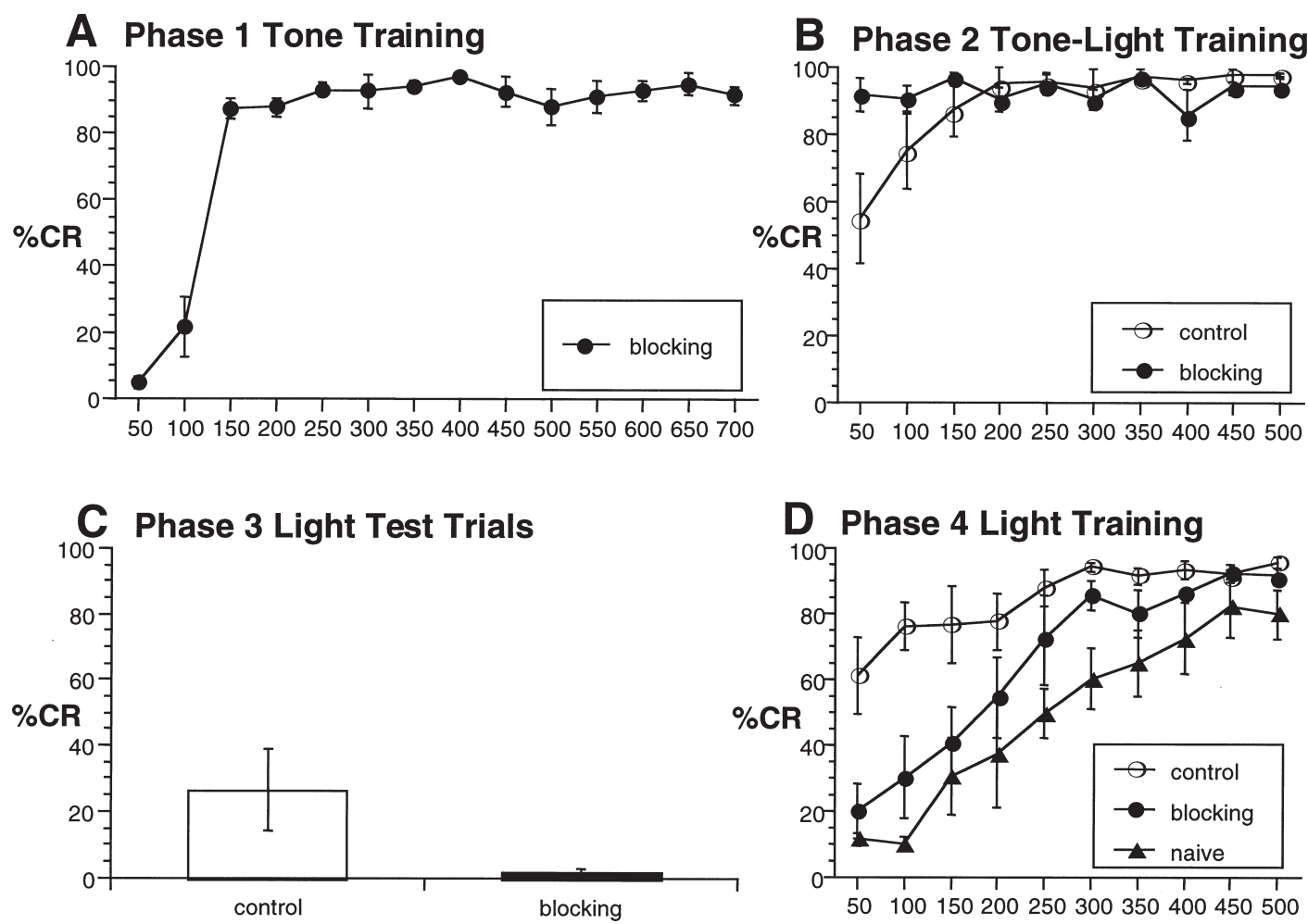

FIG. 1. shows the behavioral results four phases of the blocking experiment. Error bars represent standard error of the mean (SEM).

A. shows the learning curve for phase 1 tone-air puff training. The rabbits in the blocking group (filled squares) learned to the tone while rabbits in the control group received no stimuli.

B. shows the learning curves for phase 2 tone-light-air puff training. The rabbits in the blocking conditioning (filled squares) continued to exhibit conditioned response to the tone-light compound. Rabbits in the control group (open squares) learned to exhibit conditioned responses to the tone-light compound.

C. shows the percentage of conditioned responses for the 30 light alone test trials in phase 3 . Overall, rabbits in the blocking group exhibited fewer CRs to the light than rabbits in the control condition. This is evidence of blocking to the light.

D. shows the learning curves for the blocking (filled squares) and the control group (open squares) phase 4 light-air puff training as compared to naïve rabbits (open circles). Rabbits in the control group learned to the light faster than rabbits in the blocking conditioning. This is further evidence of blocking. Rabbits in the control group learned to the light faster than did naïve rabbits. However, rabbits in the blocking group learned at the same rate as naïve rabbits. This result does not support the hypothesis that blocking is due to learned inattention to the blocked cue.

and control groups were compared to the light training in naïve rabbits, as shown in Figure 1D. All rabbits exhibited more CRs as training progressed across sessions $(\mathrm{F}(9,108)=$ $24.168, \mathrm{p}<.001)$. There was a significant difference in conditioned responding between the three groups (blocking, control, and naïve) $(\mathrm{F}(2,12)=9.202, \mathrm{p}<.01)$. There was also a significant interaction of group $x$ session $(F(18,108)=1.907, p<.05)$, such that the rates of learning across training differed between the groups.

In pair-wise comparison, both the control group $(\mathrm{F}(1,8)=15.934, \mathrm{p}<.01)$ and the blocking group (ANOVA F $(1.7)=5.10, \mathrm{p}<.05$ ) exhibited more CRs than did the naïve 
group. For the control group and the naive group, there was a significant interaction of group $\mathrm{x}$ session $(\mathrm{F}(9,72)=3.194, \mathrm{p}<.01)$, indicating that the two groups were learning at different rates. However, for the blocking group and the naive group, there was no interaction of group $\mathrm{x}$ session (ANOVA, $\mathrm{F}(9,81)=.369, \mathrm{p}>.94$ ), indicating that the blocking and naïve groups did not differ in their learning to the light.

\section{Discussion}

Overall, the results from this blocking experiment were as expected from previous rabbit eyeblink conditioning blocking experiments (Marchant \& Moore, 1973; Kinkaide, 1974; Solomon, 1977; Kim et al., 1998). Rabbits in the blocking conditioning that had been trained to the tone followed by training to the tone-light compound exhibited significantly fewer conditioned responses to the light test trials than did controls that were only trained to the tone-light compound. In addition, rabbits in the blocking group were also slower to acquire conditioned responses to the light as compared to the control group.

Learning to the tone-light compound in the control group during phase 2 was facilitated as compared to tone alone training in the blocking group during phase 1. This facilitated learning to the tone-light compound could be due to at least two factors. First, the tonelight compound would be a more salient CS than the individual tone and light cues since it consists of a multi-modal, visual and auditory cue. Increasing CS salience is known to accelerate eyeblink conditioning (Ison \& Leonard, 1971). Prior work by Kehoe (1982) with compound stimuli in rabbit eyeblink conditioning has shown a similar pattern of facilitated learning to a tone-light compound as compared to learning to the individual components. During the first 108 training trials, rabbits receiving compound tone-light training exhibited on average about $75 \%$ CRs, rabbits trained to the tone exhibited on average about $35 \% \mathrm{CR}$, and rabbits trained to the light exhibited about $43 \%$ CRs. Therefore, it is not uncommon for compound tone-light training to produce faster acquisition than tone or light training alone.

Another factor that may have resulted in accelerated learning by the control group in phase 2 is that they sat in the chambers for seven sessions in which no stimuli were presented. This context control condition could have the effect such that the animal becomes highly attentive to the first novel event (i.e., the presentation of the tone-light compound) (Rogers et al., 2001).

We then tested the learned inattention mechanism by comparing light training for the rabbits in the blocking and control groups with that of naïve rabbits only trained to the light. Rabbits in both the blocking and control groups exhibited more conditioned responses than did the naïve rabbits. This difference is due to prior training to the light as part of the tone-light compound in Phase 2. There was no significant interaction of group $\mathrm{X}$ session for these two groups. Therefore, there was no retardation of learning from learned inattention expressed by the blocking group.

Mackintosh $(1973,1975)$ argued that learned inattention to cues involves a diminution of the associability (or "learnability") of cues such as the light in the blocking experiment- due to their being in competition with other cues that have a stronger history of being predictive of the US. Therefore, the learned inattention mechanism would predict that learning to previously blocked cue should be slower than learning by naïve subjects. Alternatively, the Rescorla-Wagner rule predicted that associative changes accruing on a trial between a CS and the US are proportional to the degree to which the US is unexpected ("surprising") given the associative strengths of all of the CS cues present on that 
trial. Therefore, since the US is perfectly predicted by the previously trained CS, there are no associative changes when the second cue is added. The error correction mechanism would predict that learning to a blocked cue would be at the same rate as learning in naïve animals. Our results for blocking in eyeblink conditioning, in which learning to blocked cue occurred at the same rate in both blocked and naïve subjects, are consistent with the prediction of the Rescorla-Wagner rule but not with the prediction of Mackintosh's learned inattention theory.

Our present experiment has a limitation based on the availability of stimuli to be used as conditioned stimuli. Other blocking studies have tested the learned inattention hypothesis by comparing the learning to the blocked CS with learning by a control group that had previously had the experience of learning to another third CS, but was naïve to the blocked cue (Mackintosh \& Turner, 1971). This control group is used so that all subjects have had the same experience of being trained to some CS prior to compound training. However, for our study in rabbit eyeblink conditioning we do not possess a well established third CS that is not an auditory (e.g., a tone, buzzer, or white noise) or visual (e.g., a light) stimulus. Recently, Disterhoft and colleagues have presented eyeblink conditioning using a tactile CS (i.e., stimulation of the vibrissae), but this CS has yet to be tested parametrically as well as the tone and light CSs. The use of a third CS that was either a tone or light would introduce the confound of generalization and transfer of associations between cues. Therefore, for our paradigm of choice, eyeblink conditioning, the comparison of a rabbit blocked to the light with rabbits that were naïve to the light is the best comparison possible. However, given this limitation, the learned inattention theory still makes a strong prediction. Acquisition to a previous blocked cue should be retarded due to learned inattention as compared to an animal that has not undergone blocking to that cue.

Another limitation of this experiment is the issue of prolonged exposure to the context in the blocking and control groups. There may be some effect in the blocked and control groups based on their prolonged exposure to the conditioning context. However, prior work in our laboratory has dealt with pre-exposure effects such as LI and LIRR (Allen, Chelius, \& Gluck (2002) and Allen et al. (2002); Shohamy et al., 2000). A control group for these pre-exposure effects is context pre-exposure in which the rabbit sits in the restraint box in the conditioning chamber for and equivalent number of blank trials in which no stimuli are presented. In these prior pre-exposure experiments, there were no significant changes in the learning curves of intact rabbits that had experience context alone pre-exposure for 450 or 850 blank trials over 4.5 or 8.5 daily sessions. In addition, recent work by Rogers et al. (2001) with LI also indicates that while a low amount of preexposures (i.e., 400 trials over 4 days) may facilitate conditioning, prolonged pre-exposure (800 trials over 8 days) to the context does not have this effect. Since the blocking and control rabbits experienced a total of 12 days of exposure to the context during the phase 1 tone training and the phase 2 tone-light training, this level of context pre-exposure would not have had any facilitation on subsequent conditioning to the light in phase 4 . It should also be kept in mind that the blocking and control rabbits did not merely experience the context for a prolonged period, but were actively learning the association between a tone or a tone-light compound and a corneal air puff. Therefore, it is unlikely that they are exhibiting any effects of prolonged contextual exposure.

Another effect of the prolonged training would be a transfer facilitation that enhanced learning to a novel CS. The tone blocking rabbits have previously learned to respond to a tone and a tone-light compound. Therefore, they may have become expert learners and could quickly acquire a CR to a novel CS (the light). However, if this was the case, the 
experience of the blocking group to learning to both the tone and tone-light compound would have resulted in a faster transfer facilitation than the tone control group that only learned to the tone-light compound. This was not the case for our results since the control group exhibited faster acquisition to the previously blocked light than did the blocking group (i.e., the normal blocking effect). Therefore, it appears that our result was due to blocking and not to pre-exposure to the context or transfer of learning from previously learned CSs to the light.

While we did not find evidence to support a learned inattention mechanism for blocking in rabbit eyeblink conditioning, we are not claiming that learned inattention is not responsible for blocking in other paradigms. Kruschke \& Blair (2000) reported that blocking in a cognitive task in humans involves learned inattention to the blocked cue. This task was an extension of the Mackintosh \& Turner (1971) finding in rats. Specifically, Kruschke \& Blair trained human subjects in a task involving disease diagnoses. They found that learning about a specific symptom was blocked by the presence of a previously trained symptom. They also found that learning about the blocked symptom is also attenuated in a later task. However, since there is a shift in the task, this data is not directly comparable to our rabbit eyeblink conditioning data that involved the same task in both the blocking and subsequent testing phases. Moreover, the Kruschke \& Blair task has a cover-story that makes the task much more cognitive than simple conditioning and therefore may involve higher brain structures such as the frontal cortex that are known to be involved in attentional and working memory tasks. Therefore, the findings of Kruschke \& Blair (2000), while different from our rabbit eyeblink conditioning results, are not directly contradictory due to differences in task demands. It would be of interest to replicate the Kruschke \& Blair study with a simple conditioning task in humans that is consistent in the blocking and subsequent testing phases or has a less complex cover-story.

One feature that may determine whether blocking is due to learned inattention or error correction is the underlying neural substrate of the specific task itself. In the case of the rabbit eyeblink conditioning results presented here, the primary learning occurs in the cerebellum and is presumed to involve an error correction mechanism like the RescorlaWagner (1972) rule (Gluck et al., 1994; Gluck et al., 2001). Therefore, it may be the case that this form of US modulation is the primary mechanism for blocking in rabbit eyeblink conditioning and not some CS modulation mechanism as proposed by others (Mackintosh, 1973; Pearce \& Hall, 1980; Kruschke \& Blair, 2000) to be responsible for blocking in other paradigms and species. However, other theories such as Schmajuk have hypothesized that US modulation (i.e., error correction) and CS modulation are co-localized within the hippocampal region. It may be that there are interactions between these two mechanisms that are not evident in eyeblink conditioning due to its reliance on cerebellar learning that is based on error correction. Future work should focus on how blocking is determined by the cerebellar and hippocampal systems known to be involved in eyeblink conditioning. It may be that blocking in eyeblink conditioning involves an interaction between error correction and some hippocampal mechanisms like that found by Baxter et al. for appetitive conditioning.

\section{References}

Allen, M.T., Chelius, L., \& Gluck, M.A. (2002). Selective entorhinal lesions and non-selective corticalhippocampal region lesions, but not selective hippocampal lesions, disrupt learned irrelevance in rabbit eyeblink conditioning. Cognitive Affective and Behavioral Neuroscience 2. 214-226.

Allen, M.T., Gluck, M.A., \& Myers, C.E. (2001). Parallel neural systems for stimulus selection in classical 
conditioning: support from computational modeling. Integrative Physiological and Behavioral Science. 36(1), 36-61.

Allen, M.T., Myers, C.E., Schnirman, G., Chelius, L., Masand, V., \& Gluck, M.A. (2002). A comparison of latent inhibition and learned irrelevance pre-exposure effects in rabbit and human eyeblink conditioning. Integrative Physiological and Behavioral Science. 37(3):188-214.

Anderson, B. J., \& Steinmetz, J.E. (1994). Cerebellar and brainstem circuits involved in classical eyeblink conditioning. Reviews in Neuroscience, 5(3), 1-23.

Baxter M.G., Gallagher, M \& Holland, P.C. (1999). Blocking can occur without losses in attention in rats with selective removal of hippocampal cholinergic input. Behavioral Neuroscience. 113. 881-890.

Chan K-H., M. J. R., Jarrard L.E., Davidson T.L. (2001). Reconsideration of the role of the hippocampus in learned inhibition. Behavioral Brain Research, 119, 111-130.

Chen, G. \& Steinmetz, J.E. (1998). A general purpose computer system for behavioral conditioning and neural recording experiments. Behavioral Research Methods, Instruments \& Computers 30:384-391.

Donegan, N. H., M. A. Gluck, \& Thompson, R.F. (1989). Integrating behavioral and biological models of conditioning. Psychology of Learning and Motivation 3, 109-156.

Donegan, N. H. \& Wagner, A. R. (1987). Conditioned diminution and facilitation of the UR: A sometimes opponent-process interpretation. Gormezano, I (Ed); Prokasy, W. F. (Ed); (1987). Classical conditioning (3rd ed.). Hillsdale, NJ, USA: Lawrence Erlbaum Associates, Inc.

Douglas, R. J. (1967). The hippocampus and behavior. Psychological Bulletin, 67(6), 416-442.

Douglas, R., \& Pribam, K. (1966). Learning and limbic lesions. Neuropsychologia, 4, 192-222.

Gluck, M.A., \& Myers, C.E. (1993). Hippocampal mediation of stimulus representation: A computational theory. Hippocampus, 3, 491-516.

Gluck, M.A., Allen, M.T., Myers, C.E., \& Thompson, R.F. (2001). Cerebellar substrates for error-correction in motor conditioning. Neurobiology of Learning and Memory. 76, 314-341.

Gluck, M.A., Myers, C.E., \& Thompson, R.F. (1994). A computational model of the cerebellum and motorreflex conditioning. An Introduction to Neural and Electronic Networks. Zornetzer, Davis, Lau and McKenna. New York, Academic Press: 91-98.

Holland, P. C. (1997). Brain mechanisms for changes in processing of conditioned stimuli in pavlovian conditioning: implications for behavior study. Animal Learning and Behavior, 25(4), 373-399.

Ison, J.R. \& Leonard, D.W. (1971). Effects of auditory stimuli on the amplitude of the nictitating membrane reflex of the rabbit (Oryctolagus cuniculus). Journal of Comparative \& Physiological Psychology. 75(1), 157-164.

Kamin, L. (1969). Predictability, surprise, attention and conditioning. Punishment and Aversive Behavior. B. Campbell and R. Church. New York, Appleton-Century-Crofts: 279-296.

Kehoe, E. J. (1982). Overshadowing and summation in compound stimulus conditioning of the rabbit's nictitating membrane response. Journal of Comparative and Physiological Psychology, 8(4), 313-328.

Kim, J. J., Krupa, D.J., \& Thompson, R.F. (1998). Inhibitory cerebello-olivary projections and blocking effect in classical conditioning. Science, 279, 570-573.

Kim, J. J., \& Thompson, R.F. (1997). Cerebellar circuits and synaptic mechanisms involved in classical eyeblink conditioning. Trends in Neuroscience, 20, 177-181.

Kinkaide PS. (1974) Stimulus selection in eyelid conditioning in the rabbit (Oryctolagus cuniculus). Journal of Comparative and Physiological Psychology 86:1132-1140.

Kruschke, J.K., \& Blair, N.J. (2000). Blocking and backward blocking involve learned inattention. Psychonomic Bulletin \& Review. 7, 636-645.

Mackintosh, N. (1973). Stimulus selection: Learning to ignore stimuli that predict no change in reinforcement. In R. Hinde \& J. Stevenson-Hinde (Eds.) Constraints on Learning: Limitations and Predispositions (pp. 75-96). New York: Academic Press.

Mackintosh, N. J. (1975). A theory of attention: Variations in the associability of stimuli with reinforcement. Psychological Review, 82, 276-298.

Mackintosh, N. J., \& Turner, C. (1971). Blocking as a function of novelty of CS and predictability of UCS. Quarterly Journal of Experimental Psychology, 23, 359-366.

Marchant III, H. G., \& Moore, J.W. (1973). Blocking of the rabbit's conditioned nictitating membrane response in kamin's two-stage paradigm. Journal of Experimental Psychology, 101, 153-158.

Martin, I., \& Levey, A.B. (1991). Blocking observed in human eyelid conditioning. The Quarterly Journal of Experimental Psychology, 43B, 233-256.

Pearce, J. and G. Hall (1980). “A model for Pavlovian learning: Variations in the effectiveness of conditioned but not of unconditioned stimuli." Psychological Review 87, 532-552.

Rescorla, R. and A. Wagner (1972). A theory of Pavlovian conditioning: Variations in the effectiveness of 
reinforcement and non-reinforcement. Classical Conditioning II: Current Research and Theory. A. Black and W. Prokasy. New York, Appleton-Century-Crofts: 64-99.

Rogers, R.F., Katz, D.B., Gorin, R.E., \& Steinmetz, J.E. (2001). Contextual contributions to the expression of latent inhibition. Society for Neuroscience Abstracts.

Sears, L. L., \& Steinmetz, J.E. (1991). Dorsal accessory inferior olive activity diminishes during acquistion of the rabbit classically conditioned eyelid response. Brain Research, 545, 114-122.

Shohamy, D., Allen, M.T., \& Gluck, M.A. (2000). Dissociating entorhinal and hippocampal function in latent inhibition. Behavioral Neuroscience. 114, 867-874.

Solomon, P.R. (1977). Role of the hippocampus in blocking and conditioned inhibition of the rabbit's nictitating membrane response. Journal Comparative Physiological Psychology. 91, 407-417.

Solomon, P. R. (1980). A time and place for everything? temporal processing views of hippocampal function with special reference to attention. Physiological Psychology, 8(2), 254-261.

Thompson, L.T., Moyer, J.R., Akase, E., \& Disterhoft, J.F. (1994). A system for quantitative analysis of associative learning. Part 1. Hardware interfaces with cross-species applications. Journal of Neuroscience Methods. 54, 109-117. 
Copyright $\odot 2003$ EBSCO Publishing 